\title{
Valeur diagnostique des différentes formes parasitaires dans la Trypanosomose américaine expérimentale en immuno-fluorescence chez la Souris
}

\author{
par M.-A. POTHIER, G.A. SCHMUÑIS, N.-H. VATTUONE, \\ O. C. TRAVERSA et J.-F. YANOVSKY \\ (Instituto de Microbiologia y Parasitologia, Facultad de Medicina, Paraguay 2155, \\ Universidad de Buenos-Aires - Argentina - Professor A.S. PAROdI)
}

\section{Résumé}

L'Immuno-fluorescence indirecte a permis de détecter les anticorps contre les formes de culture et contre les formes sanguines de Trypanosoma cruzi chez la souris, et d'effectuer simultanément une comparaison.

Il n'a pas été observé de différences significatives des taux pour les différentes formes parasitaires.

\section{Summary}

Indirect immuno-fluorescence was used to detect the antibodies against culture and blood forms of Trypanosoma cruzi, and to make the comparison simultaneously.

No significant differences in titrage have been observed for the different parasitic forms.

\section{Resumen}

Se analiza en este trabajo la utilidad de la Inmunofluorescencia como procedimiento para la detección de anticuerpos contra formas sanguineas del Tripanosoma cruzi en el raton ; comparándola simultanemente con el mismo método aplicado a crithidias.

No se observa diferencias significativas de titulos para las distintas formas parasitarias. 


\section{Introduction}

Depuis que la réaction d'immuno-fluorescence a été appliqué au diagnostic des Protozooses, elle s'est montrée comme une excellente méthode de diagnostic (1) (2).

En ce qui concerne la Trypanosomose américaine, son utilisation diagnostique remonte à plusieurs années, bien que sa spécificité soit encore à l'étude, on considère cette technique comme sensible et précoce (3).

Nous basant sur l'expérience acquise pour d'autres applications, nous avons recherché les anticorps anticrithidias (formes de culture) et les anticorps antitrypanosomes (formes sanguines) dans la Trypanosomose américaine expérimentale chez la souris, par la technique d'immuno-fluorescence indirecte.

\section{Matériels et Méthodes}

a) LES ANTIGÈNES.

Les Crithidias utilisées proviennent de la souche Tulahuen cultivées sur milieu biphasique, dont la composition est comparable à celle décrite dans des travaux antérieurs (4).

Les Trypanosomes de souche Tulahuen proviennent du sang de souris $\mathrm{C} 3 \mathrm{H}$ pesant 15 grammes, infectées sept jours auparavant avec $1 \times 10^{6}$ Trypanosomes virulents, et saignées par section de la veine axillaire.

Les Crithidias sont lavées trois fois dans du sérum physiologique et remises en suspension dans la même solution, formolée à $1 \%$ à la température ambiante, pendant 30 minutes, pour les fixer. Ensuite, elles sont à nouveau lavées trois fois dans du sérum physiologique.

Les Trypanosomes provenant du sang de souris rendu incoagulable par un excès d'héparine (1.000 U.I. d'héparine par $\mathrm{cm}^{3}$ de sang) sont séparés des globules rouges par centrifugation à 1.200 tours par minute, pendant deux minutes. Le surnageant est constitué par le plasma qui contient les Trypanosomes et les plaquettes.

A partir du culot, on répète deux fois l'opération, en ramenant au volume initial à l'aide de la solution de Eagle, dans le but d'extraire la plus grande quantité de Trypanosomes.

Ultérieurement, les plaquettes sont détruites par agitation avec des billes de verre. Les Trypanosomes obtenus sont soumis au même traitement que celui décrit pour les Crithidias.

Après le dernier lavage par le sérum physiologique, le culot antigénique est remis en suspension dans du tampon à $\mathrm{pH} 7,2$ jusqu'à l'obtention d'une concentration de cinq parasites par champ, avec un objectif à immersion, et des oculaires $\times 8,5$.

On utilise des lames microscopiques en verre ordinaire de $0,8 \mathrm{~mm}$ d'épaisseur, dont une face est au préalable quadrillée avec du vernis synthétique pour permettre de réaliser simultanément dix dilutions par lame.

On dépose une goutte de suspension antigénique au centre de chaque case, puis on sèche les lames dans une étuve à $37^{\circ} \mathrm{C}$. 
Une conservation de l'ordre de cinq à six mois, est assurée en plaçant les lames au congélateur à $-20^{\circ} \mathrm{C}$.

\section{b) LES SÉRUMS ÉTUDIÉs.}

Les souris utilisées sont de la souche Balb/C.

Les Crithidias de la souche Tulahuen proviennent de milieux de culture biphasiques, avec plus de 60 passages en culture et $1,4 \%$ de formes métacycliques.

Quand les souris avaient 25 jours, nous leur avons inoculé par voie intrapéritonéale $1 \times 10^{6}$ Crithidias de culture au troisième jour. Ces Crithidias avaient été préalablement lavées trois fois dans du sérum physiologique. La quantité de Crithidias a été déterminée à l'aide d'une cellule de Neubauer.

Les souris ont été sacrifiées au $3^{\circ}, 7^{\circ}, 10^{\circ}, 15^{\circ}, 20^{\circ}, 25^{\circ}, 28^{\circ}$ et $30^{\circ}$ jour après l'infestation par les Crithidias. Les sérums ont été recueillis après section de la veine axillaire, puis conservés à $-20^{\circ} \mathrm{C}$.

L'étude de chaque prélèvement porte sur cinq souris au moins.

Nous avons retenu comme dilution initiale le $1 / 10$ dans du tampon à $\mathrm{pH} 7,2$.

Nos sérums ont été étudiés aux dilutions $1 / 10,1 / 20,1 / 40, \ldots 1 / 5120$. Dans chaque série de sérums étudiés, on inclut deux sérums témoins ; un témoin positif et un témoin négatif, tous les deux dilués au 1/10.

c) Le CONJUgué.

Nous avons utilisé un sérum fluorescent de lapin anti-globulines de souris de l'Institut Pasteur *, dilué dans du tampon à $\mathrm{pH} 7,2$. La dilution au $1 / 10$ du conjugué a été déterminée face à un sérum positif provenant d'une souris immunisée (5), et face à un sérum négatif.

\section{d) CONTre-Coloration.}

On utilise du bleu d'Evans (R.A.L.) dilué à 1/10.000 suivant la technique décrite par P. Ambroise-Thomas (6) pour la Toxoplasmose et par M.-A. Pothier (7) pour la Bilharziose.

\section{e) INSTALlATION MiCROSCOPIQUE.}

Nous utilisons un microscope Zeiss, avec un condensateur à fond clair, à sec. Objectifs $\times 16, \times 40$. Oculaires $\times 8,5$.

La source lumineuse est constituée par une lampe à brûleur à vapeur de mercure (Osram HBO 200) alimentée par un appareil stabilisateur de tension. Filtre d'émission BG 12/3 mm. Filtre d'arrêt 53 Zeiss.

Grâce à de petites œillères de caoutchouc, disposées sur les oculaires, il est possible de travailler dans une pièce normalement éclairée.

* Nous remercions M. le Professeur J. Coudert, à l'amabilité duquel nous devons ce sérum. 


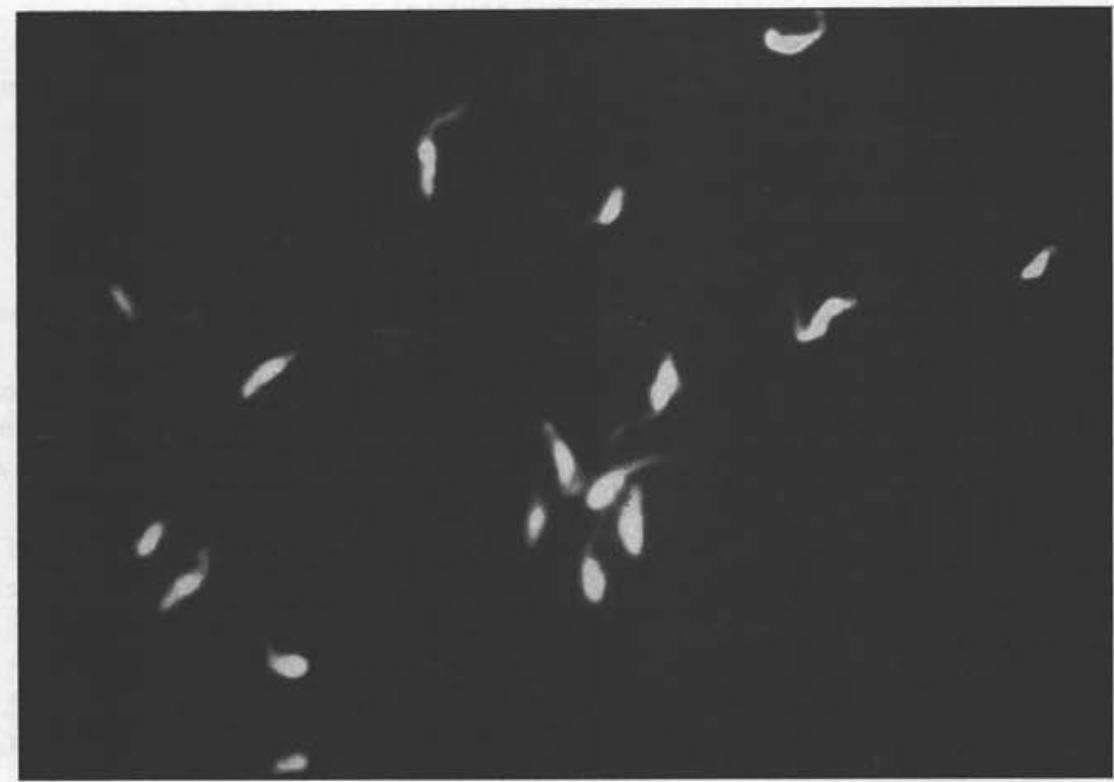

Рното $\mathrm{n}^{\circ}$ 1. - Séro-diagnostic de la maladie de Chagas sur la forme crithidias. Réaction positive (1) (Les éléments présentent une vive fluorescence jaune-verte).

(1) Pour nos photographies, nous avons utilisé des pellicules Agfacolor CK. Temps de pose 10 minutes. Objectif $\times 40$. Oculaires $\times 12,5$.

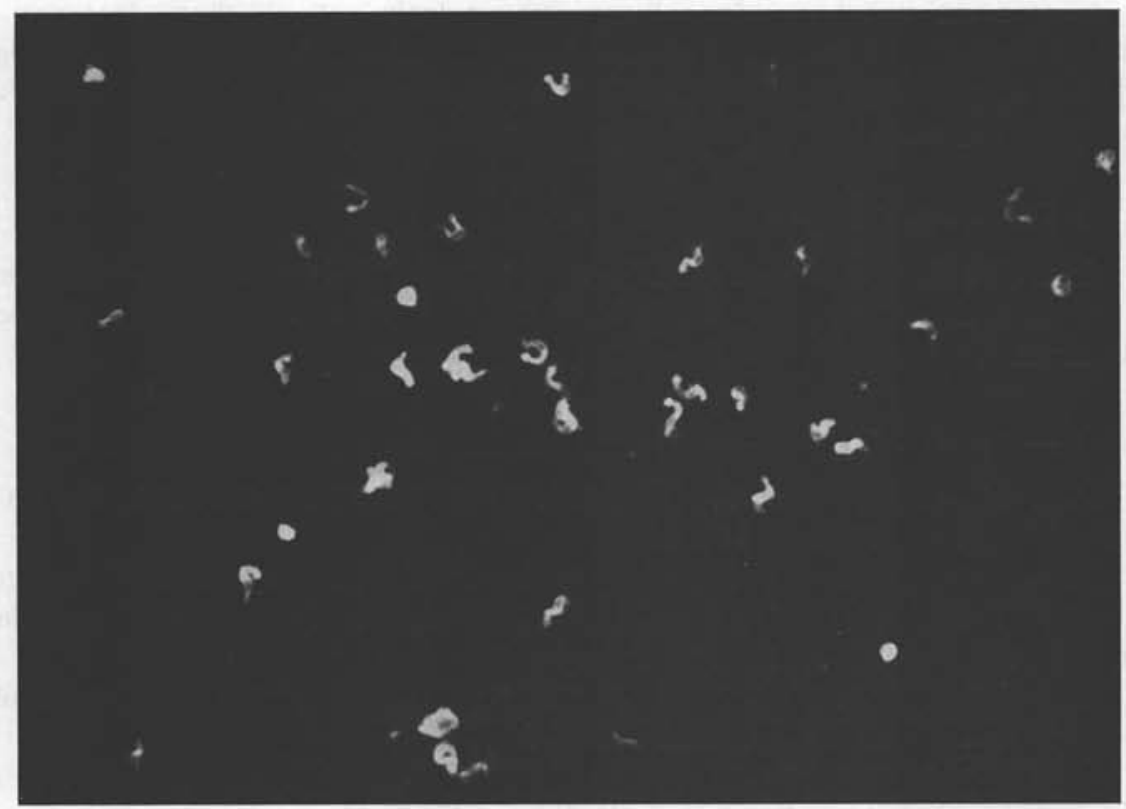

Рното $\mathrm{n}^{\circ}$ 2. - Séro-diagnostic de la maladie de Chagas sur la forme trypanosome. Réaction positive (Fluorescence jaune-verte spécifique). 


\section{Résultats.}

Grâce à la contre-coloration qui élimine les réactions douteuses, la lecture des résultats est nette et rapide.

Quand la réaction est positive, les Crithidias (Photo 1) et les Trypanosomes (Photo II) présentent une vive fluorescence jaune-verte.

Lorsque la réaction est négative, les parasites présentent une coloration rouge sombre uniforme.

Le taux des anticorps anti-crithidias 1/40 au quinzième jour augmente progressivement jusqu'au $1 / 1280$ au trentième jour.

Les anti-corps anti-trypanosomes par ailleurs, apparaissent aussi au quinzième jour pour atteindre un maximum de 1/640 au vingt-huitième jour (Tableau I).

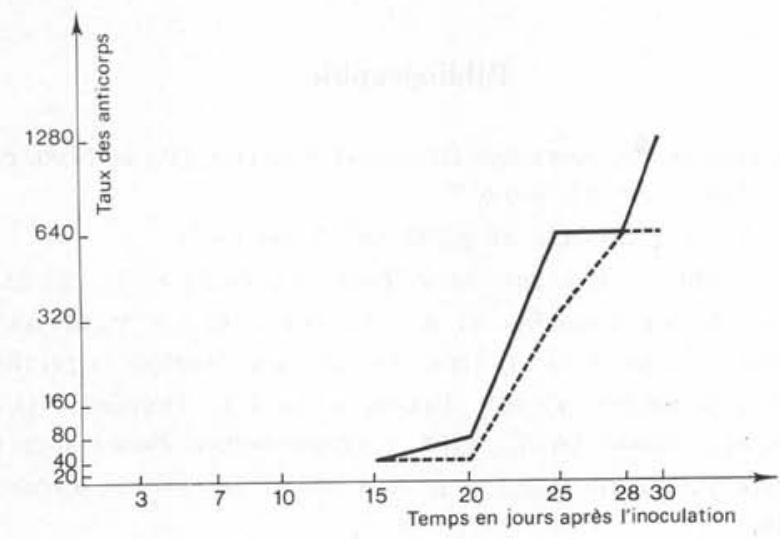

\section{TABLEAU I}

Evolution des anticorps fluorescents chez des souris infectées au $25^{\mathrm{e}}$ jour par $1 \times 10^{6}$ crithidias

- anticorps anticrithidias.

- - anticorps antitrypanosomes.

\section{Discussion.}

L'immuno-fluorescence indirecte a démontré être une méthode utile pour l'évaluation des anticorps chez la souris, face à différents stades évolutifs de Trypanosoma cruzi.

Jusqu'à présent l'existence de sérieuses difficultés pour extraire les antigènes à partir des formes sanguines de Trypanosoma cruzi, ont empêché d'étudier avec précision la réponse immunologique à ce stade du parasite. L'immuno-fluorescence a remédié à cet inconvénient grâce à la faible quantité d'antigène nécessaire. 
Les sérums des souris inoculées par les formes de culture (Crithidias), produisent une réponse immunologique assez comparable mis en présence des deux formes du parasite.

Ceci peut être dû :

a) A l'existence d'antigènes superficiels communs pour les deux stades,

b) Ou au fait que les anticorps contre les formes de culture sont dûs à l'inoculation des Crithidias (98,5 \% de l'inoculat) et que les anticorps anti-trypanosomes auraient pour origine, l'infection active produite par les Trypanosomes métacycliques, qui sont encore présents en faible proportion dans la culture inoculée.

Ce travail préliminaire, sera complété par l'étude des autres formes parasitaires, notamment par les formes tissulaires et par les formes provenant de l'hôte intermédiaire.

\section{Bibliographie}

1. Coudert (J.), Garin (J.-P.), Ambroise-Thomas (P.), Saliou (P.) et Minjat (M.), 1965. Bull. Soc. Path. exot. 58, 630-639.

2. Cornet (A.). - Thèse Pharmacie. 86 p., 82 réf. (Lyon 1967).

3. Camargo (M. E.), 1966. - Rev. Inst. Med. Trop., São-Paulo 8 (5), 227-234.

4. Gonzalez Cappa (S. M.), Schmuñis (G. A.), Traversa (O. C.), Yanovsky (J. F.), Parodi (A. S), 1968. - Journal of Infections Diseases and Hygiene (à paraître).

5. Yanovsky (J.F.), Schmuñis (G. A.), Taratuto (A. L.), Traversa (O. C.), Gonzalez CAPPA (S. M.), PAROdi (A. S.), 1968. - Experimental Parasitology (à paraître).

6. Ambroise-Thomas (P.), Garin (J.-P.) et Rigaud (A.), 1966. - La Presse Médicale 74, 2215-2216.

7. Pothier (M.-A.), 1967. - Thèse Médecine, 160 p., 185 réf. (Paris). 